with those of Evans and Montgomery, prove incontestably that the ligature of the carotid only may arrest the development, not only of aneurisms of the innominate, but also of those of the arch of the aorta." (See page 108 of this volume of THe LANCET.)

But I am quite ready to admit all the merit which belongs to the practical enforcement of what was at best only a vague suggestion; and nothing could be further from my intention than to pass over too lightly a case which I regard as one of the most interesting and important in the modern surgery of aneurism.

With regard to the discussion at the Medical Society of London, reported in your last, on the way in which this distal operation acts, I thought I had succeeded in showing that in cases at least where no branch intervenes between the ligature and the sac, the distal ligature of any of the arteries opening out of it tends to obliterate and fill with clot that part of the aneurismal sac through which the current used to pass into the artery now obliterated. And I produced preparations and drawings which appeared to me to prove the precise similarity of this result, obtained artificially by the distal ligature, with the result of the natural impaction of clot in the arteries leaving the sac, which is so frequent a phenomenon in aneurism, and which Mr. Guthrie long ago alluded to as an effort of nature towards the cure of the disease.

If this view be correct, the ligature of the left carotid would check the growth of an aneurism of the arch of the aorta in those cases in which the sac is growing at the part acted on by the stream of blood passing up the mouth of the carotid. The operation would be plainly indicated in cases where the tumour conld be traced as making progress up the left side of the neck towards the left sternoclavicular joint. This is a point which is difficult to decide in any given case (and the difficulty must be admitted as an objection to the operation), but the dilatation of the left pupil, the congestion of the left eye, the comparative feebleness of the left radial pulse, and the dyspnca complained of when the patient lay with the left side uppermost, seem to testify in Dr. Cockle's case to an extension of the disease latterly towards the left side, it having begun nearer the origin of the arch. And the immediate relief from these symptoms, which the patient experienced from the stoppage of eirculation through the left carotid, seems to me to prove that the coagulation which the ligature would produce in the lower part of the artery extended into the aneurismal sac, and consolidated this portion of it. We know that this effect often, if not invariably, follows on the impaction of clot. How can we doubt that the same effect may be produced by the ligature?

$$
\text { Yours truly, }
$$

Clarges-street, Nov. 1872.

T. HoLMES.

\section{COPPER IN THE HUMAN BODY.}

\section{To the Editor of The LANCET.}

Srr, - - In your remarks last week on the case Reg. $v$. Kettle, you very properly call attention to the existence of copper in the human body, but at the same time imply that this fact did not receive due attention from myself, the analyst employed by the prosecution.

It is not possible to glean from a newspaper all the medical and chemical evidence offered in such an important case as this. May I therefore be allowed to point out what amount of attention the special point to which $I$ have reFerred received, and to indicate an important fallacy that underlies an ordinary search for copper?

The stomach of Mrs. Kettle, as well as the liver, yielded indubitable evidence of the presence of copper. I have examined many other human stomachs for copper, but never detected the metal except when there was no doubt of the administration of copper.

Twelve ounces of the liver yielded one-fifteenth of a grain of metallic copper, equal to more than $a$ quarter of a grain of blue vitriol. I have never heard of such an amount being met with as an accidental constituent of a human liver; and, speaking from the results of numerous analyses of my own, made with special reference to the existence of copper in that viseus, I think it unlikely that such a quantity of copper would be met with had there been no administration of the metal as a medicine or poison.
In my evidence I laid considerable stress on the existence of copper in the human body, and also in articles of food and drink, adducing the alleged fact that copper had been found in wheat and other plants grown in Essex, the county in which the woman died.

My own researches, and those of other chemists also have recently thrown great doubt on the supposed general existence of copper in the body and in common articles of food and drink. I find that it is scarcely possible to incinerate any animal or vegetable substance over an ordinary gas-burner or spirit lamp without transferring portion of the copper of the brass used in the construction of the lamp to the ash of the substance incinerated. Where special precautions are not taken to avoid this source of error, no reliance can be placed upon the results. In my analyses in connexion with this case $I$ had lamps specially constructed of steatatite and iron to meet this difficulty. A great number of articles of food and drink, from sources as nearly as possible identical with those employed by the deceased, were also analysed. In none of them was any copper detected, except in black tea, and then in insignificant quantities.

I am, Sir, your obedient servant,

Caversham-road, Nov. 12th, 1872.

Thomas Stevenson.

\section{INQUESTS WITHOUT POST-MORTEM EXAMINATIONS.}

To the Editor of The LANCET.

SIR,-I feel that the circumstances I am about to lay before you are of so much public importance that I need offer no apology for asking you to devote a portion of your valuable space to their consideration.

During the afternoon of Thursday, October 24th, a man was brought into this institution by the police in a state of insensibility. The police found him in a public urinal early in the morning, and a surgeon who saw him recommended his removal to the infirmary. The man could not be identified, and had nothing whatever in his pockets when he arrived here. His right eye was destroyed from old disease, and the left pupil was firmly contracted. His clothes were soiled with what appeared, and smelt like, vomited matter. Respiration was quite regular, but accompanied with vibrations of the buccinator muscles. The body was much cooler than natural, and the pulse very weak and feeble. Behind the left ear was a recent scratch, an inch long, which was bleeding, and the face and neck upon the same side were much congested. No other mark indicating violence could be discovered. The stomach was washed out with warm water, which returned coloured with bile only. There was no smell of drink or opium to be distinguished in the fluid upon its return from the stomach. The urine was drawn off, and found free from albumen. Finding that the man's condition did not improve, and that he seemed to be dying, it was considered right to inject hypodermically five minims of a solution of atropine, on the theory of the possibility of opium having been taken. The solution was specially prepared for this case by Mr. Brady, the President of the Pharmaceutical Society, and contained the one-hundredth part of a grain of atropine in five minims of fluid. No indication of physiological action could be ascertained to follow the injection and it was not repeated. The man died in the night. An inquest was held, and the surgeon who saw the case previous to its admission was summoned. I sent a resident clinical clerk, an advanced student, to the inquest, with instructions that he should inform the jury there were not sufficient grounds on which to return a verdict without an internal examination of the body. The coroner did not order any post-mortem, and upon the opinion of the surgeon whom he had summoned, a verdict was given that serous apoplexy, the result of drink and exposure, was the cause of death. Accidentally an opportunity was afforded me of seeing the skull opened, when it was discovered there was an extensive fracture of the internal plate of the occipital bone on the left side, with fissures extending in various directions. The right lobe of the brain was extensively and the left slightly lacerated, and clotted blood was effused over the surface of the brain. What other internal injuries this man may have sustained I do not know.

I acquainted the coroner with the fact that the man had 